\title{
Radiosensibilité de rameaux greffons d'abrico- tier exposés à un rayonnement gamma aigu et observation en pépinière d'une deuxième généra- tion végétative de bourgeons irradiés
}

Jean-Michel LEGAVE \& Gilbert GARCIA

E.N.S.A.-I.N.R.A., Laboratoire d'Arboriculture fruitière, 9, place Viala, F 34060 Montpellier Cedex

RÉSUMÉ

\begin{abstract}
L'amélioration ou la diversification de la gamme variétale demeure une nécessité pour le maintien ou la relance de la culture de l'abricotier en France. Dans cette optique un programme de mutagenèse induite par irradiation de bourgeons a été envisagè. Une connaissance approfondie de la radiosensibilité du matériel végétal choisi a été une première étape indispensable, en raison notamment du peu de données bibliographiques à ce sujet. Ainsi des rameaux greffons de différents cultivars d'abricotier ont été soumis à des doses croissantes de rayonnement gamma à fort débit. Les effets physiologiques radioinduits d'ordre quantitatif sont estimés selon des critères couramment utilisés (taux de survie, croissance). Ces résultats constituent des éléments de base pour situer dans un premier temps la dose "optimale » de traitement pour des conditions relativement bien définies (stade dormant des bourgeons, débit de dose de 6 à $7 \mathrm{~Gy} / \mathrm{mn}$ ). Des scions de deuxième génération végétative (V2) ont été constitués afin d'observer, en pépinière puis en verger, les effets génétiques provoqués par les différents traitements effectués. On devrait ainsi mieux déterminer la dose optimale de traitement et, plus généralement, mieux appréhender l'intérêt de la méthode utilisée. Les observations effectuées en pépinière font déjà apparaître les limites de cette méthode.
\end{abstract}

Mots clés additionnels : Amélioration variétale, mutagenèse induite, arbre fruitier.

Radiosensitivity of apricot budsticks exposed to acute gamma rays and nursery observations on the second vegetative generation from irradiated buds.

Varietal improvement is essential if a new impetus is to be given to the French apricot crop. We have accordingly started a mutation breeding programme by bud irradiation. Because of the scarcity of bibliographical data, the first step was to study the budwood radiosensitivity of the apricot cultivars used as starting material. Budsticks of various cultivars were exposed to increasing doses of acute gamma rays. The physiological effects induced by irradiation were evaluated from bud survival and growth. These results allowed us, in the first instance, to fix the optimal dose range for dormant buds irradiated at 6 to $7 \mathrm{~Gy} / \mathrm{mn}$. Observations in the nursery on scions from the second vegetative generation (V2) already showed the limits of bud irradiation. Further observations in the orchard are need to estimate the value of this method for apricot breeding.

Additional key words : Varietal improvement, induced mutations, fruit tree.

\section{INTRODUCTION}

Pour les arbres fruitiers, l'exposition à un rayonnement gamma de bourgeons dormants est une méthode classique de création variétale par mutagenèse induite. Cette méthode vise à améliorer ou à diversifier, pour un ou quelques caractères, des cultivars ayant déjà une bonne valeur agronomique. Dans ce domaine l'abricotier n'a semble-t-il fait l'objet que de quelques travaux à l'étranger (BROERTJES \& VAN HARTEN, 1978).
L'élargissement et l'amélioration de la gamme variétale apparaissent pourtant depuis longtemps comme des facteurs déterminants pour l'avenir de cette culture, notamment en France (COURANJOU, 1975). Par ailleurs, cette méthode, appliquée aux principaux cultivars, pourrait s'avérer particulièrement appropriée au cas de l'amélioration variétale de l'abricotier. Ces cultivars sont en effet des variétés anciennes souvent étroitement adaptées à une région de culture et difficiles à remplacer, notamment pour des raisons d'adapta- 
tion, par des cultivars introduits ou créés par hybridation.

La mise en œuvre d'un programme de création variétale par irradiation de bourgeons nécessite tout d'abord une bonne connaissance de l'influence de la dose de traitement sur la survie et la croissance du matériel végétal utilisé dans les conditions de traitement retenues. Ainsi dans un premier temps il est généralement nécessaire d'étudier les effets physiologiques de différentes doses car ces derniers peuvent largement varier suivant les espèces ou les cultivars, le stade d'évolution des bourgeons lors du traitement et enfin les conditions de traitement, notamment le débit de dose (LAPINS et al., 1969 ; VISSER, 1973 ; BROERTJES \& VAN HARTEN, 1978 ; LAPINS, 1983). Nous rapportons ici des résultats d'une telle étude dans le cas de l'abricotier compte tenu du peu de données bibliographiques concernant la radiosensibilité des bourgeons chez cette espèce (LAPINS, 1983). Ces résultats sont complétés par des observations en pépinière sur de jeunes arbres issus de cette étude préliminaire.

\section{MATÉRIELS ET MÉTHODES}

Des irradiations aux rayons gamma de cobalt 60 ont été pratiquées 2 années de suite au Centre d'Etudes Nucléaires de Cadarache. On a utilisé un appareil de type « gamma-cell » dont la source émet un rayonnement à débit de dose relativement élevé puisqu'ayant été d'environ $7 \mathrm{Gray} / \mathrm{mn}$ la première année et $6 \mathrm{~Gy} / \mathrm{mn}$ la deuxième année $(1 \mathrm{~Gy}=100 \mathrm{rad})$. Les irradiations ont été effectuées la première année à 2 dates différentes et à doses relativement modérées ( 20 et $30 \mathrm{~Gy}$ puis 20,30 et $40 \mathrm{~Gy}$ ) compte tenu du niveau élevé du débit de dose et de données bibliographiques situant la D.L. 50 (dose de rayonnement induisant une létalité des bourgeons de 50 p. 100 par rap- port au taux de survie d'un échantillon témoin) pour l'abricotier. Les résultats obtenus à la suite de ces premières irradiations ont conduit l'année suivante à étudier les effets d'une gamme de doses plus élevée $(30,50$ et $70 \mathrm{~Gy}$ ). Cinq cultivars ont été irradiés la première année (10 greffons/dose). Il s'agit de «Rouge du Roussillon », "Polonais », "Bergeron », «Précoce de Tirynthe " et «Beliana ». Seuls ces 2 derniers ont été de nouveau irradiés l'année suivante (20 greffons/ dose). Les 3 premiers sont de vieilles variétés françaises, étant chacune assez étroitement adaptée à une région de culture et assurant ensemble, encore actuellement, l'essentiel de la production française. "Précoce de Tirynthe " est une variété introduite de Grèce. L'origine génétique de ces 4 cultivars est inconnue ; ce sont vraisemblablement des semis de hasard issus d'autofécondation (autogamie préférentielle des cultivars européens). Le cultivar «Beliana» a par contre une origine hybride bien connue puisqu'il est issu d'un croisement contrôlé entre un cultivar local tunisien (phylum Nord-Africain) et le cultivar «Canino » d'origine espagnole.

La figure 1 résume la méthode suivie avant et après irradiation des rameaux greffons. Dans tous les cas des rameaux ont été prélevés puis irradiés au stade bourgeons dormants. Des rameaux portant des bourgeons plus évolués (début de débourrement) ont été également irradiés pour le cultivar «Beliana » lors de la deuxième année d'expérimentation. L'évolution des greffons après surgreffage a fait l'objet de notations classiques afin d'estimer les effets physiologiques radioinduits. Par la suite de jeunes arbres de deuxième génération végétative (scions $V_{2}$ ) ont été constitués et observés en pépinière pour déceler dès ce stade d'éventuelles mutations. On peut noter que contrairement à des travaux analogues (DECOURTYE \& LANTIN, 1971), un double écussonnage a été effectué afin de mieux rentabiliser les travaux de pépinière mais aussi pour augmenter les chances d'isolement de secteurs mutés étroits et éventuellement préciser la filiation entre les bourgeons greffés (fig. 1).

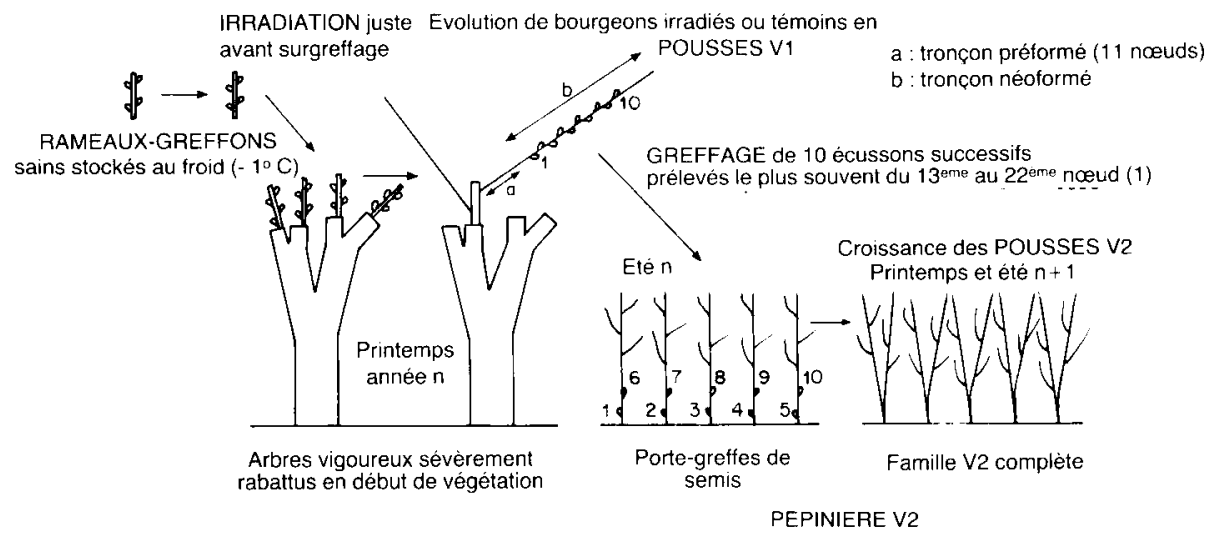

(1) Recherche des mutations situèes dans le méristème terminal du

bourgeon irradié, compte tenu des observations de DECOURTYE et LANTIN (1971) 


\section{RÉSULTATS}

\section{A. Radiosensibilité des rameaux greffons}

\section{Taux de survie des rameaux greffons (fig. 2a)}

Ce taux est clairement fixé environ un mois après le surgreffage. Les greffons ne survivant pas se dessèchent en ne présentant aucun signe de croissance. Dans le cas des traitements de la première année, l'amplitude réduite de la gamme de doses et le peu de greffons par traitement conduisent à présenter un taux de survie pour l'ensemble des cultivars. Il apparaît ainsi une sensible diminution de ce taux à partir de $30 \mathrm{~Gy}$ pour les 2 dates d'irradiation (entre 20 et 30 p. 100 de la survie des témoins). Les résultats de l'année suivante montrent également une diminution du taux de survie à $30 \mathrm{~Gy}$, bien que d'importance variable suivant le cultivar ou le stade des bourgeons. Par contre, 70 Gy apparaît comme une dose létale ou sub-létale quel que soit le matériel traité. Une dose intermédiaire de $50 \mathrm{~Gy}$ semble également induire une diminution très importante du taux de survie comme le suggère le résultat obtenu pour «Beliana " au stade dormant. Par ailleurs on peut noter des taux de survie assez variables entre les différents témoins. Ainsi outre l'irradiation, un greffage tardif et plus encore un début d'évolution des bourgeons apparaissent comme des facteurs diminuant le taux de survie.

\section{Taux de bourgeons ayant évolué en pousses $V_{l}$} (fig. 2b)

Ce taux, calculé par rapport au nombre initial de bourgeons, traduit l'influence d'un traitement à la fois sur la survie des greffons et sur l'aptitude des bourgeons des greffons demeurés vivants à évoluer en pous- ses de première génération végétative $\left(\mathrm{V}_{1}\right)$. Le nombre de ces pousses est fixé assez rapidement. L'évolution de ce taux en fonction de la dose de traitement est dans l'ensemble très voisine de celle du taux de survie des greffons (fig. 2a et b). Seule l'irradiation à $30 \mathrm{~Gy}$ de «Beliana » au stade dormant s'est traduite par une diminution significative du nombre de pousses $V_{1}$ sur les greffons demeurés vivants. On peut donc penser que les méristèmes primaires (apex) et secondaires (cambium) ont des radiosensibilités du même ordre.

La dose induisant une diminution de 50 p. 100 du taux de pousses $V_{1}$ de l'échantillon témoin peut être assimilée à la notion de D.L. 50 couramment utilisée pour estimer les dommages physiologiques. Pour des bourgeons irradiés au stade dormant les divers résultats obtenus conduisent à la situer entre 30 et $50 \mathrm{~Gy}$ (probablement plus près de $50 \mathrm{~Gy}$ dans la plupart des cas). Cette dose pourrait être plus ou moins élevée suivant le cultivar, comme le suggère la plus grande sensibilité à 30 Gy de «Beliana » par rapport à « Précoce de Tirynthe » (deuxième année d'expérimentation).

\section{Somme des longueurs des pousses $V_{l}$ (fig. 2c)}

Ce dernier critère intègre à la fois le critère précédent et les effets bien connus de lenteur de la croissance après irradiation. Des mesures de longueur des pousses $V_{1}$ à une date donnée n'ont été effectuées que pour les traitements de la deuxième année. Environ un mois et demi après le surgreffage une importante diminution de la somme des longueurs des pousses $V_{1}$ a été observée dès la dose de $30 \mathrm{~Gy}$ pour les 3 matériels traités. Pour " Précoce de Tirynthe ", ceci s'explique essentiellement par une diminution de la longueur moyenne des pousses $V_{1}$ (significative et de près de 30 p. 100). Pour «Beliana » (stade dormant), la diminution de la somme des longueurs à $30 \mathrm{~Gy}$ apparaît nettement plus importante. Elle s'explique en effet à la fois par une

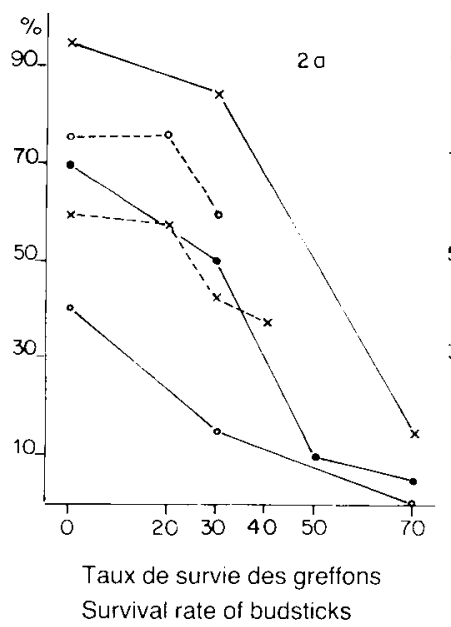

Figure 2

Influence de la dose d'irradiation sur la survie et l'évolution des bourgeons en pousses de première génération végétative $\left(V_{l}\right)$.

Effect of irradiation dose on the survival and growth of primary shoots $\left(V_{1}\right)$.

Première année d'irradiation (tous cultivars confondus).

First year of irradiation (all the cultivars mixed).

-..-o 25 avril (april 25).

$\mathrm{x}-\ldots \mathrm{x} 16$ mai (may 16).
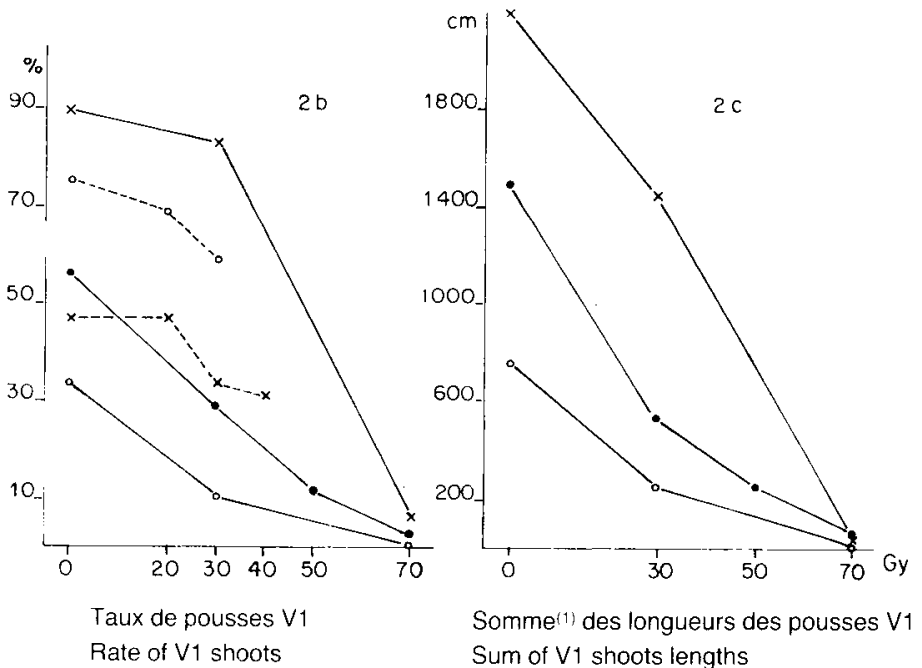

Somme ${ }^{11}$ des longueurs des pousses $V 1$ Sum of $V 1$ shoots lengths

(1) Mesures effectuées à $\pm 0,5 \mathrm{~cm}$. 44 jours après le surgreffage Measurements at $+0,5 \mathrm{~cm}, 44$ days after grafting

Deuxième année d'irradiation (23 avril).

Second year of irradiation (april 23).

$\mathrm{x}$.... $\mathrm{x}$ «Précoce de Tirynthe ».

- - «Beliana », bourgeons dormants (dormant buds).

느 "Beliona», bourgeons en début de débourrement theginning of buds growth). 
diminution de la longueur moyenne des pousses (significative et de près de 30 p. 100 également) et par une diminution de la survie des bourgeons précédemment indiquée.

\section{Manifestations qualitatives}

Très peu d'effets physiologiques radioinduits d'ordre qualitatif ont été observés : quelques cas de malformations foliaires ou de fasciation sur la pousse $V_{1}$.

\section{B. Effets génétiques observés en pépinière $V_{2}$}

\section{Importance et nature des observations}

Sur 2 ans un peu plus de 1500 scions ont été observés dont environ 10 p. 100 de témoins, chaque scion étant constitué d'1 ou 2 pousses $V_{2}$ suivant la réussite du double écussonnage (plus de 50 p. 100 de scions à 2 pousses). Ce matériel correspond à 312 familles issues d'irradiation, comportant 4 ou 5 scions pour la plupart. La réussite de l'écussonnage est apparue sans relation avec les doses d'irradiation appliquées aux bourgeons à l'origine des pousses $V_{1}$. Seules, des observations qualitatives ont été effectuées. Des notations quantitatives n'ont pas été envisagées pour diverses raisons techniques (hétérogénéité initiale des porte-greffes, scions à 1 ou 2 pousses $V_{2}, \ldots$ ).

\section{Type, fréquence et localisation des mutations}

Aucune mutation de réduction de vigueur (port « compact ") n'a été décelée, alors que ce type de mutation peut être observé sans mensuration tant au stade $V_{1}$ qu'en pépinière $V_{2}$ (DECOURTYE \& LANTIN, 1971 ; VISSER, 1973). Les mutations décelées ne concernent que la forme et la coloration des feuilles : plus petites, plus allongées, lacérées, gaufrées, et relativement souvent panachées (déficience chlorophyllienne). Seulement 3,8 p. 100 des familles issues d'irradiation, soit 12 familles, ont présenté de telles mutations. Ce taux global ne recouvre aucune différence marquée suivant le cultivar ou la dose d'irradiation. D'autre part pour toutes ces familles, les mutations sont apparues assez localisées puisque n'ayant été observées que sur 1 ou 2 scions pour 11 de ces familles et sur 3 scions pour une panachure visible dès le stade $V_{1}$. Dans plusieurs cas d'un unique scion affecté, seules certaines ramifications sur une même pousse $V_{2}$ ont présenté des mutations du feuillage. Ces observations indiquent que dans tous les cas, la lignée cellulaire mutante occupait une position étroite, voire très étroite, dans le méristème terminal du bourgeon initial après irradiation. La localisation des mutations suivant le rang du bourgeon greffé peut s'interpréter différemment suivant les cas observés : phyllotaxie sur la pousse $V_{1}$ de $2 / 5$, éventuellement de $3 / 8$, avec élimination possible des cellules mutées au cours de l'ontogenèse (sélection diplontique). Pratiquement pour 9 des familles mutantes, le greffage des bourgeons de rangs 1 à 5 aurait suffit pour isoler le secteur muté.

\section{DISCUSSION ET CONCLUSION}

D’une façon générale des doses croissantes de rayonnement provoquent une diminution de la survie et inversement une augmentation de la fréquence des mutations avec un risque de plus en plus élevé que des mutations favorables soient accompagnées de mutations défavorables, ce qui conduit à la notion de dose optimale de traitement (BROERTJES \& VAN HARTEN, 1978). En réalité de multiples facteurs interfèrent pour déterminer l'importance des effets physiologiques et génétiques radioinduits, certains de ces facteurs étant difficilement contrôlables (état physiologique des bourgeons et conditions de croissance après irradiation). Pour des traitements ultérieurs de plus grande ampleur il semble donc préférable de traiter les bourgeons à différentes doses susceptibles d'avoir un effet optimal.

Dans un premier temps cette gamme de doses peut être située en fonction des effets physiologiques observés. Une réduction de survie des bourgeons de 50 p. 100 (D.L. 50) est un critère souvent retenu, mais l'emploi de doses à effets plus modérés est également conseillé afin de limiter la fréquence des mutations défavorables (VISSER, 1973 ; BROERTJES \& VAN HARTEN, 1978 ; LAPINS, 1983). Par ailleurs des doses modérées (plus de 50 p. 100 de survie) permettent d'associer des taux élevés de survie et de mutations (LAPINS et al., 1969 ; Silvy \& MitTEAU, 1986). Les résultats obtenus pour l'abricotier conduisent à situer la D.L. 50 entre 30 et 50 Gy pour des bourgeons « dormants » exposés à un rayonnement aigu. DONINI (1975; in LAPINS, 1983) situe cette dose à 40 Gy pour des conditions comparables de traitement. Dans l'optique d'une sélection de mutants directement utilisables, il semble judicieux, compte tenu des remarques précédentes, de retenir pour des traitements de plus grande ampleur des doses de 30 à 40 Gy ayant induit des effets physiologiques modérés.

Notons que l'abricotier semble se caractériser par une relative sensibilité des méristèmes secondaires, généralement plus radiorésistants que les méristèmes primaires. Des doses de 50 et 70 Gy ont en effet provoqué un dessèchement total d'une très grande proportion de greffons, contrairement à ce que l'on observe chez le pommier (DECOURTYE \& LANTIN, 1971) ou chez les agrumes (HEARN, 1986).

Les observations en pépinière laissent entrevoir les limites de la méthode utilisée. Tout d'abord il faut souligner le faible taux de familles mutantes observé à ce stade par rapport à celui indiqué pour le pommier par DECOURTYE \& LANTIN (1971). Ceci pourrait résulter de différences quant à l'importance des doses d'irradiation, au rang des bourgeons prélevés sur les pousses $\mathrm{V}_{1}$ ou à la précision des observations. Toutefois l'abricotier semble être une espèce relativement stable comme le suggèrent l'apparente rareté des mutants naturels et des travaux antérieurs (LAPINS, 1973 ; LEGAVE, 1985). Cette relative stabilité pourrait s'expliquer en partie par un niveau relativement faible d'hétérozygotie, notamment pour les variétés anciennes. De même il faut souligner la localisation étroite, voire très étroite, des mutations observées. Cet inconvénient classique a pu être accentué par le niveau généralement faible ou modéré des doses de traitement à l'origine de 
ces mutations (LAPINS et al., 1969). Pour des traitements ultérieurs à doses modérées, le double écussonnage pratiqué en pépinière $V_{2}$ paraît donc intéressant puisque les chances d'isolement de mutations étroites sont probablement augmentées sans augmentation du nombre de porte-greffes par famille. Cette technique limite par contre les possibilités de criblage des mutations de vigueur, qui ne sont toutefois pas très recherchées chez l'abricotier. En définitive plusieurs années d'observation en verger du matériel obtenu devraient permettre de mieux apprécier l'intérêt des traitements effectués et par conséquent de préciser la méthode à utiliser pour l'obtention d'un matériel de plus grande ampleur.

Reçu le 21 janvier 1987. Accepté le 17 septembre 1987.

\section{REMERCIEMENTS}

Nous tenons à remercier $\mathrm{A}$. SIIVy pour ses conseils et la réalisation des irradiations au Service de Radio-Agronomie du C.E.N. de Cadarache. Ces remerciements s'adressent également à $\mathrm{P}$. CROSSARAYNAUd et J. M. Audergon pour leur aide et l'accueil de nos essais sur les domaines de la Station de Recherches Fruitières Méditerranéennes du centre I.N.R.A. de Montfavet.

\section{RÉFÉRENCES BIBLIOGRAPHIQUES}

Broertjes C., Van Harten, 1978. Application of mutation breeding methods in the improvement of vegetatively propagated crops. Elsevier Scientific Publishing Company, Amsterdam, $316 \mathrm{p}$.

Couranjou J., 1975. L'amélioration génétique de l'Abricotier par l'I.N.R.A., Bull. tech. Inf. Min. Agric., $1^{\circ}$ et $2^{\circ}$ partie, 300, 391-400, $3^{\circ}$ partie, $301,469-482$.

Decourtye L., Lantin B., 1971. Considérations méthodologiques sur l'isolement de mutants provoqués chez le Pommier et le Poirier. Ann. Amélior. Plantes, 21 (1), 29-44.

Hearn C. J., 1986. Development of seedless grapefruit cultivars through budwood irradiation. J. Amer. Soc. Hort. Sci., 111 (2), 304306.

Lapins K. O., 1973. Induced mutations in fruit trees. 1-19, in Induced mutations in vegetatively propagated plants, Vienna, IAEA.

Lapins K. O., 1983. Mutation breeding. 74-99, in Methods in fruit breeding edited by Moore J. N. and Janick J. : Purdue University Press, West Lafayette, Indiana, 464 p.

Lapins K. O., Bailey C. H., Hough L. F., 1969. The effects of gamma rays on apple and peach buds at different stages of development. I. Survival, growth and mutation frequencies. Radiat. Bot., 9, 379-389.

Legave J. M., 1985. Comparaison de la variabilité de populations d'abricotiers issus de graines irradiés et non irradiés de même origine. Agronomie, 5 (5), 445-453.

Silvy A., Mitteau Y., 1986. Diversification des variétés d'oeillet par traitement mutagène. 395-405, in Nuclear techniques and in vitro culture for plant improvement, Vienna, IAEA.

Visser T., 1973. Methods and results of mutation breeding in deciduous fruits, with special reference to the induction of compact and fruit mutations in apple 21-33, in Induced mutations in vegetatively propagated plants, Vienna, IAEA. 